American Journal of Applied Sciences 7 (12): 1550-1557, 2010

ISSN 1546-9239

(C) 2010 Science Publications

\title{
The Role of Sine and Cosine Components in Love Waves and Rayleigh Waves for Energy Hauling during Earthquake
}

\author{
Zainal Abdul Aziz and Dennis Ling Chuan Ching \\ Department of Mathematics, Faculty of Science, \\ University Technology Malaysia, 81310 Johor, Malaysia
}

\begin{abstract}
Problem statement: Love waves or shear waves are referred to dispersive SH waves. The diffusive parameters are found to produce complex amplitude and diffusive waves for energy hauling. Approach: Analytical solutions are found for analysis and discussions. Results: When the standing waves are compared with the Love waves, similar cosine component is found in both waves. Energy hauling between waves is impossible for Love waves if the cosine component is independent. In the view of 3D particle motion, the sine component of the Rayleigh waves will interfere with shear waves and thus the energy hauling capability of shear waves are formed for generating directed dislocations. Nevertheless, the cnoidal waves with low elliptic parameter are shown to act analogous to Rayleigh waves. Conclusion: Love waves or Rayleigh waves are unable to propagate energy once the sine component does not exist.
\end{abstract}

Key words: Energy hauling, sine and cosine, amplitude, love waves, Rayleigh waves, Cnoidal waves, earthquake, phase velocity, periodic, isotropic medium, illustrated, anisotropic

\section{INTRODUCTION}

Seismic body waves are classified to $\mathrm{P}, \mathrm{SH}$ and $\mathrm{SV}$ waves. Similar waves have being derived in vector form (Ari-Menahem and Singh, 1981). These waves will give the surface Rayleigh waves and Loves waves that decay exponentially with depth. Rayleigh waves and Love waves are recognized for near surface displacement and there are categorized under dispersive waves since the phase and group velocities are different (Bhatnagar, 1979). Tsunami waves or long waves are also the surface waves. However, these waves are dissimilar with Rayleigh waves or Love waves (Ribal, 2007; 2008).

Standing waves are known as stationary waves that do not propagate. There arise in a motionless medium as a result of interference involving two waves traveling in opposite directions. Similar incidence can be explained through the movement along the tectonic plate where the interference of waves is invincible. The capability of standing waves to propagate forward has attracted researchers' interest. In experimental study, (Brothers et al., 1996) attributed the standing waves from systematic fractures such as vein structures during seismic wave shaking. Later, Tsuneo and Yujiro (Ohsumi and Ogawa, 2008) found vein structures that are formed by shear waves during earthquake. In seismology, Love waves are referred to shear waves and $\mathrm{SH}$ waves in layered medium are similar to Love waves. For this reason, the similarity between standing waves and Love waves must be exploited. Complexity of fractures and Love waves must be explained to resolve the mechanism of vein structures formation especially the direction of dislocation.

Standing waves and progressive waves are different in general. Standing waves do not involve in the form of energy and momentum transfer between waves separated by nodes while progressive waves are propagating the energy. Since Rayleigh waves are near surface waves, multi layer mediums are not suitable for showing near surface displacement. However, the splitting shear waves or SV waves from Rayleigh waves have affected the Rayleigh wave's dispersive behavior for layered anisotropic medium (Zhang et al., 2009). The statement for splitting SV waves is progressive in layered medium must be explained. Energy hauling capability should be considered as well.

Sine and cosine components in progressive waves and standing waves explain the different between Rayleigh and Love waves. Seismic waves are periodic waves and thus the displacement has a periodic variation with time and distance. Despite being periodic, conidial waves have sharper crest and flatter troughs than sinusoidal waves. Usually, these waves are

Corresponding Author: Zainal Abdul Aziz, Department of Mathematics, Faculty of Science, University Technology Malaysia, 81310 Johor, Malaysia 
referred to ocean waves which are distinguished by the elliptic parameter. When the elliptic parameter reduces to zero, smooth repetitive oscillations are formed and these waves are similar to seismic waves. Both waves should look alike at medium surface if the antinodes of the waves are ignored. Eventually, it must be explained the Rayleigh wave has a possibility to transform into Cnoidal waves once the elliptic parameter is increasing to produce nonlinear seismic waves that can propagate energy. Apparently, nonlinear waves are known for self reinforcing and capable of propagating vast energy in the medium (Dauxious and Peyrard, 2006; Drazin and Johnson, 1989).

\section{MATERIALS AND METHODS}

Problem statement: Seismic waves are divided into two groups $\mathrm{P}$ and $\mathrm{S}$ in according to the propagation direction. The $S$ waves again are categorized into two sub groups SV and SH waves. The explanations for seismic waves in energy hauling and 3D particle motion are more remarkable if these waves are featured in sine and cosine components.

The combination of the Loves waves and Rayleigh waves will give the $3 \mathrm{D}$ particle motion as shown in Fig. 1. P, SV and SH waves introduced by BenMenahem and Singh (1981) read:

$$
\begin{aligned}
& \mathrm{U}_{\mathrm{P}}=\mathrm{A}\left(\mathrm{la}_{\mathrm{x}}+\mathrm{na} \mathrm{a}_{\mathrm{z}}\right) \exp \left[\mathrm{iw}\left(\mathrm{t}-\frac{\mathrm{lx}+\mathrm{nz}}{\alpha}\right)\right] \\
& \mathrm{U}_{\mathrm{SV}}=\mathrm{B}\left(-\mathrm{na}_{\mathrm{x}}+\mathrm{la} \mathrm{a}_{\mathrm{z}}\right) \exp \left[\mathrm{iw}\left(\mathrm{t}-\frac{\mathrm{lx}+\mathrm{nz}}{\beta}\right)\right] \\
& \mathrm{U}_{\mathrm{SH}}=\mathrm{Ca}_{\mathrm{y}} \exp \left[\mathrm{iw}\left(\mathrm{t}-\frac{1 \mathrm{x}+\mathrm{nz}}{\beta}\right)\right]
\end{aligned}
$$

Where:

1 and $\mathrm{n} \quad=$ The vector components

$\mathrm{a}_{\mathrm{x}}, \mathrm{a}_{\mathrm{y}}$ and $\mathrm{a}_{\mathrm{z}}=$ Refer to the unit vector

$\alpha$ and $\beta=$ The velocity for $\mathrm{P}$ and $\mathrm{S}$ waves

$\mathrm{A}, \mathrm{B}$ and $\mathrm{C}=$ Refer to the amplitude of the waves with finite depth

$\mathrm{z}$ and $\mathrm{w} \quad=$ The frequency of the waves

For elliptic particle motion, similar frequency with respect to the wave is expected. It is appropriate to reduce (1), (2) and (3) through the relations that read:

$\mathrm{n}_{\delta}=\sqrt{\frac{\mathrm{c}^{2}}{\delta^{2}}-1}, \mathrm{c}=\frac{\delta}{1}$

$\frac{\mathrm{lx}+\mathrm{nz}}{\delta}=\frac{\mathrm{x}+\mathrm{nz} / 1}{\delta / 1}=\frac{\mathrm{x} \pm\left(\sqrt{\frac{\mathrm{c}^{2}}{\delta^{2}}-1}\right) \mathrm{z}}{\mathrm{c}}$
Where:

$$
\begin{array}{ll}
\mathrm{C} & =\begin{array}{l}
\text { The velocity of the phase } \\
\delta=\alpha \text { or } \beta
\end{array}=\begin{array}{l}
\text { The velocity of the } \mathrm{P} \text { or } \mathrm{S} \text { waves } \\
\text { respectively } \\
\mathrm{K}
\end{array} \\
\eta_{\delta}=\eta_{\alpha} \text { or } \eta_{\beta}= & \text { Refers to the wavenumbers }
\end{array}
$$

Inserting the relations (4) and (5) into equations (1), (2) and (3), we will get the dispersive form of seismic waves as:

$$
\begin{aligned}
& \mathrm{U}_{\mathrm{P}}=\mathrm{A}\left(\mathrm{a}_{\mathrm{x}} \pm \mathrm{n}_{\alpha} \mathrm{a}_{\mathrm{z}}\right) \exp \left[\mathrm{ik}\left(\mathrm{ct}-\mathrm{x} \mp \eta_{\alpha} \mathrm{z}\right)\right] \\
& \mathrm{U}_{\mathrm{SV}}=\mathrm{B}\left(\mathrm{a}_{\mathrm{z}} \mp \mathrm{n}_{\beta} \mathrm{a}_{\mathrm{x}}\right) \exp \left[\mathrm{ik}\left(\mathrm{ct}-\mathrm{x} \mp \eta_{\beta} \mathrm{z}\right)\right] \\
& \mathrm{U}_{\mathrm{SH}}=C \mathrm{Ca}_{\mathrm{y}} \exp \left[\mathrm{ik}\left(\mathrm{ct}-\mathrm{x} \mp \eta_{\beta} \mathrm{z}\right)\right]
\end{aligned}
$$

The combination of Eq. 6 and 7 will form the Rayleigh waves while Eq. 8 alone is known as Love waves (Ben-Menahem and Singh, 1981). These dispersive waves will decay exponentially with finite depth.

For the case of $\delta>c$, we get the complex $\eta_{\delta}$. Inserting the complex $\eta_{\delta}$ to either Eq. 6-8, the selected Eq. 8 reads:

$$
\mathrm{U}_{\mathrm{SH}}=\operatorname{Cexp}\left(i \eta_{\beta} \mathrm{z}\right) \exp [\mathrm{ik}(\mathrm{ct}-\mathrm{x})]
$$

After linear polarization with respect to $\mathrm{y}$-direction. $\mathrm{C}$ Is the amplitude while $\exp \left(i \eta_{\beta} z\right)$ is referring to the diffusive term. Apparently, Eq. 9 is non diffusive since the term $\exp \left(i \eta_{\beta} z\right)$ is in complex (Bhatnagar, 1979).

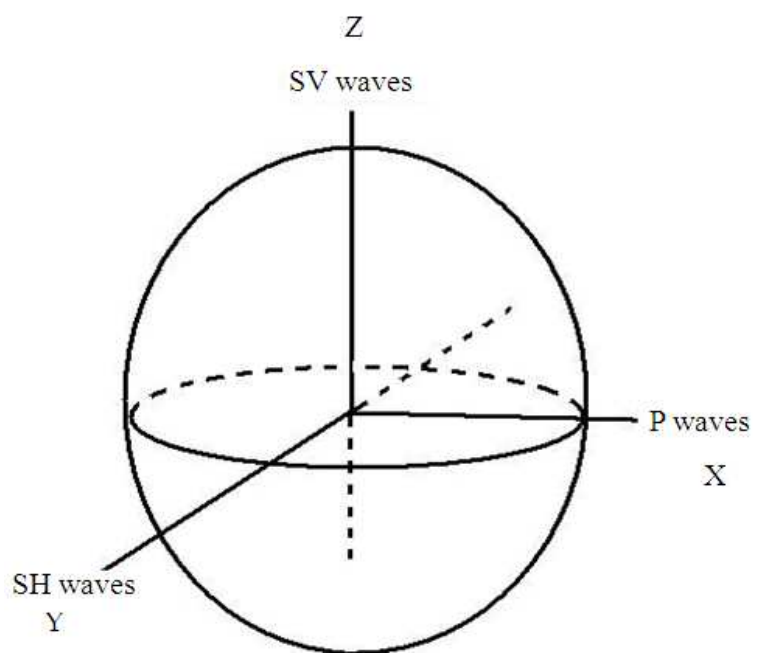

Fig. 1: The seismic wave's framework 
Am. J. Applied Sci., 7 (12): 1550-1557, 2010

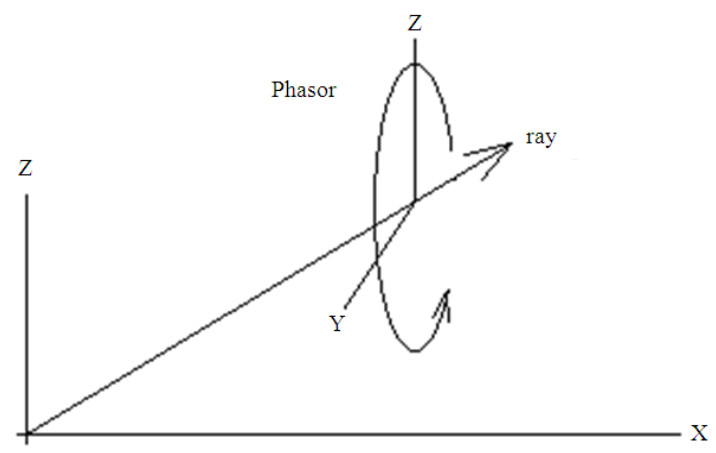

Fig. 2: The amplitude and diffusion behavior of the $\mathrm{S}$ waves
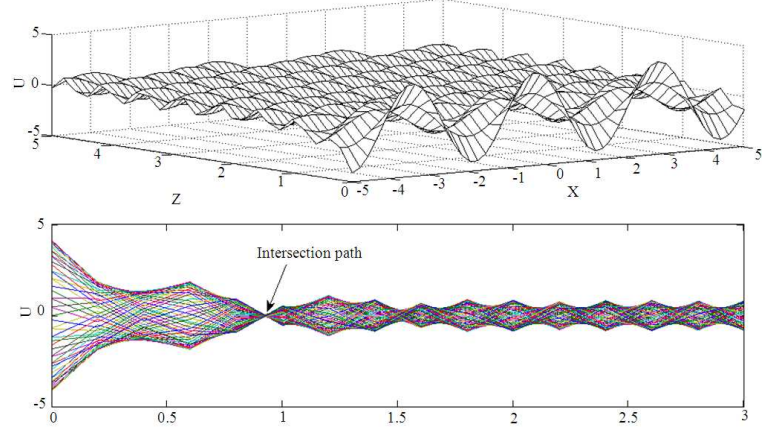

Fig. 3: The interference of $\mathrm{S}$ waves

From Eq. 6-8, $\eta_{\alpha}$ and $\eta_{\beta}$ give the dispersive waves with amplitude $\mathrm{A}, \mathrm{B}$ and $\mathrm{C}$. The explanation can go into diffusive characteristic of the waves and also the complex amplitudes.

Complex amplitude is usually referred to phasor or amplitude of the waves. Figure 2 illustrates the propagation behavior of the $\mathrm{SV}$ waves featuring complex amplitude. It is possible for SV waves become $\mathrm{SH}$ waves after polarization. Conforming to the rotating behavior, $\mathrm{S}$ wave is plotted for the dispersion and diffusion behaviors as illustrated in Fig. 3. S waves have dispersed into many and diffused with depth $\mathrm{z}$. These $S$ waves are not featuring energy hauling behavior. In the following, the justifications will be shown.

Love waves cosine component: For finite layer of depth, the Love waves are referred to the Eq. 8 . According to Fig. 1 the waves move in both positive and negative $y$ direction as:

$\mathrm{U}_{\mathrm{SH}}=\mathrm{a}_{\mathrm{y}}\left[\mathrm{C}_{1} \exp \left(-\mathrm{ik} \eta_{\beta} \mathrm{z}\right)+\mathrm{C}_{2} \exp \left(\mathrm{ik \eta} \eta_{\beta} \mathrm{z}\right)\right] \exp [\mathrm{ik}(\mathrm{ct}-\mathrm{x})]$

The induced stress by the propagating Love waves in the medium cannot be ignored. By using the stress components introduced by Ben-Menahem and Singh (1981), the stress read:

$$
\begin{aligned}
\tau_{32}= & \mu \mathrm{u}_{2,3}=\mu_{1}\left[\mathrm{C}_{1} \exp \left(-\mathrm{i} \eta_{\beta} \mathrm{kz}\right)+\mathrm{C}_{2} \exp \left(\mathrm{i} \eta_{\beta} \mathrm{kz}\right)\right] \\
& \exp [\mathrm{ik}(\mathrm{ct}-\mathrm{x})] \\
\tau_{32}= & \mu \mathrm{u}_{2,3}=\mu_{1} \exp [\mathrm{ik}(\mathrm{ct}-\mathrm{x})]
\end{aligned}
$$

where, $\mathrm{u}_{1}, \mathrm{u}_{2}, \mathrm{u}_{3}$ are in $\mathrm{x}, \mathrm{y}, \mathrm{z}$ directions. Equation 11 shows the stress within the layered medium while the free surface stress is depicted through Eq. 12 when $\mathrm{z}=0$. Despite the absent of complex amplitude and diffusive term, the periodic sinusoidal waves are still exist.

Love waves are usually explained in a layer over a half space medium. There is significant different between free surface stress and $\tau_{32}$ stress. Stress is also recognized as diffusion energy. Free surface has less energy at the free surface whereas $\tau_{32}$ stress produces radical stress in close proximity to the surface featuring complex or non amplitude as illustrated in Fig. 4a and $4 \mathrm{~b}$ respectively.

Equation 10 can be written in cosine form with amplitude $\mathrm{C}_{1}=\mathrm{C}_{2}$ that reads:

$\mathrm{U}_{\mathrm{SH}}=\mathrm{Ca}_{\mathrm{y}} \cos \left(\eta_{\beta} \mathrm{kh}\right) \exp [\mathrm{ik}(\mathrm{ct}-\mathrm{x})]$

Equation 13 shares the similarity with the standing waves (Bhatnagar, 1979).

According to (Bhatnagar, 1979), the standing waves do not include in the form of energy and momentum transfer between waves separated by nodes. This explains Love waves do not forward energy. Since Love waves only contribute to the cosine component, this kind of waves are considered as non moving waves which vibrate at the origin without participating in energy transformation. Conforming to the definition of standing waves, the capability of Love waves in energy hauling is non-existence. Fig. 3: illustrates the intersection path of the Love waves featuring the interference of the wave moving in opposite direction to diminish the energy and momentum transfer between nodes.

The stress component between 2 layers can be written as:

$$
\begin{aligned}
& \mathrm{C}_{1}\left(-\exp \left(-\mathrm{i} \eta_{1} \mathrm{kh}\right)+\exp \left(\mathrm{i} \eta_{1} \mathrm{kh}\right)\right)= \\
& \frac{\left(\eta_{2} \mu_{2}\right)}{\left(\eta_{1} \mu_{1}\right)} \mathrm{C}_{2}\left(-\exp \left(-\mathrm{i} \eta_{2} \mathrm{kh}\right)+\exp \left(\mathrm{i} \eta_{2} \mathrm{kh}\right)\right)
\end{aligned}
$$




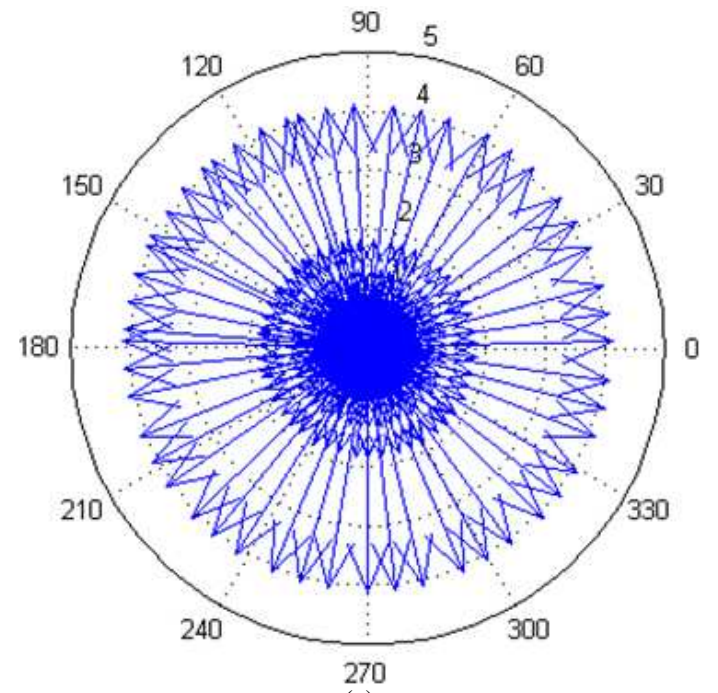

(a)

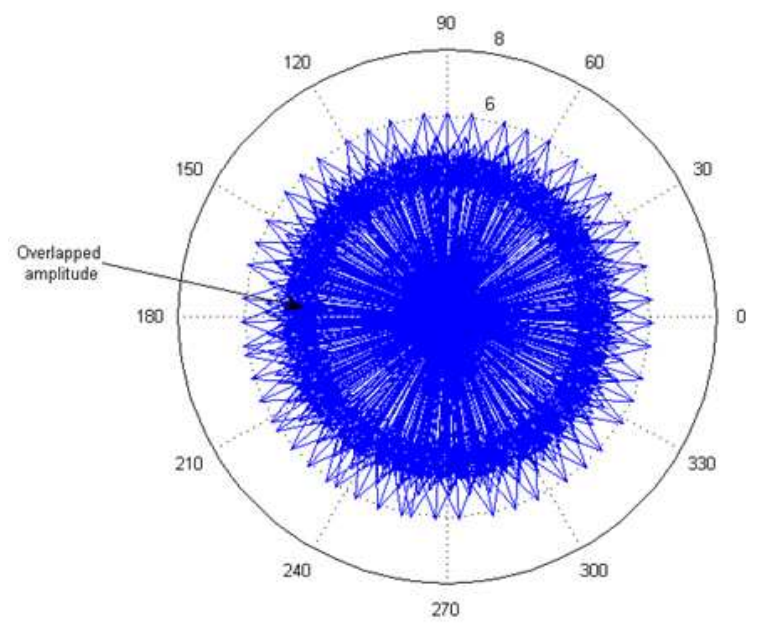

(b)

Fig. 4: (a) The interference of the $S$ waves limiting the energy from being brought forward. (b) The $\mathrm{S}$ waves with high diffusion energy at the epicenter. (b) The overlapped amplitude or diffusion energy near the medium surface

It is recognized in isotropic medium, there exists two kinds of waves: pressure waves and shear waves. The two diffusion parameters in 2 layers of isotropic medium are expected to give two kinds of shear waves. From Eq. 14, $\mu_{1}$ and $\mu_{2}$ are the shear modulus or modulus of rigidity of the medium. When this is applied to Love waves, the shear modulus and the density $\rho$ give the velocity of shear wave $V_{s}$ that reads:

$$
\mathrm{V}_{\mathrm{s}}=\sqrt{\mu / \rho}
$$

where the shear modulus of waves are related to shallowness' parameter such that the shear stress are proportional to the shallow of the layered medium. Letting $A=C_{1}, B=C_{2}$ et a $1=\eta_{1}$ and et a $2=\eta_{2}$ in Fig. 5, the complex diffusive parameter are examined for the energy diffusion and Love wave dispersion in two layered medium. It is observed that the displacement will be greater at layer 2 according to the depth of the stress distributed by Love waves. The amplitudes of the Love waves in two different layers of medium are seen to generate nearly equal stress at both directions when both the amplitudes $\mathrm{C}_{1}$ and $\mathrm{C}_{2}$ are nearly similar. In fact, the medium density for two layers of medium must be different.

In the same way Eq. 14 can transform into cosine form that reads:

$$
\begin{aligned}
& A \cos \left(\eta_{1} k h\right) \exp [i k(c t-x)]= \\
& B \cos \left(\eta_{2} k h\right) \exp [i k(c t-x)]
\end{aligned}
$$

In the following, the sine and cosine components are studied to show the role of sine and also the cosine components.

Rayleigh waves sine and cosine components: Rayleigh waves and Love waves should not share the same diffusion given that Rayleigh waves are guided near surface waves while Love waves are guided shear waves in a layered isotropic medium. Free surface stress should not consider the shallowness of the medium such that a single layered isotropic medium is discussed for Rayleigh waves instead of two layered medium.

In previous following, Love waves propagate as standing waves since the cosine component exists in $\mathrm{SH}$ waves. However, the Love waves which are also the shear waves either SV or SH waves are progressive if there are independent. In the view of 3D particle motion, these shear waves will interfere with the $P$ waves.

For the case mentioned by Zhang et al. (2009), the vein structure or the displacement is brought by the shear waves that propagate within the medium. Hence, it is well said the P-SV waves which are also the Rayleigh waves must show some characteristic of $S$ waves. It can be shown that the SV waves of the Rayleigh waves which are also the shear waves are generating stress to the medium and the stress is brought forward by another part of the Rayleigh waves or P waves. In other words, the Rayleigh waves involve in the form of energy and momentum transfer between waves separated by nodes. 
In this study, energy is said being forwarded by the sine component of the Rayleigh waves from the view of 3D particle motion by the elliptic waves. These elliptic seismic waves are built up by $\mathrm{P}, \mathrm{SV}$ and $\mathrm{SH}$ waves as illustrated in Fig. 1. The explanation can go into diffusion energy or stress when the Rayleigh waves are proven for carrying sine and cosine components.

Rayleigh waves are built from $\mathrm{P}$ and SV waves from (6) and (7) that reads:

$$
\mathrm{U}_{\mathrm{P}}+\mathrm{U}_{\mathrm{SV}}=\left[\begin{array}{l}
\mathrm{A}\left(\mathrm{a}_{\mathrm{x}}+\mathrm{i} \eta_{\alpha} \mathrm{a}_{\mathrm{z}}\right) \exp \left(-\eta_{\alpha} \mathrm{kz}\right)+ \\
\mathrm{B}\left(\mathrm{a}_{\mathrm{z}}+\mathrm{i} \eta_{\beta} \mathrm{a}_{\mathrm{x}}\right) \exp \left(-\eta_{\beta} \mathrm{kz}\right)
\end{array}\right] \exp [\mathrm{ik}(\mathrm{ct}-\mathrm{x})]
$$

Ben-Menahem and Singh (1981) introduces the stress components for $\mathrm{P}$ and $\mathrm{S}$ waves. The $\mathrm{P}$ waves stress components read:

$\tau_{31}=2 \mu \mathrm{u}_{1,3}, \tau_{33}=\lambda \mu_{1,1}+(\lambda+2 \mu) \mathrm{u}_{3,3}$

While the $\mathrm{S}$ waves stress component read:

$\tau_{31}=\mu\left(\mathrm{u}_{1,3}+\mathrm{u}_{3,1}\right), \tau_{33}=2 \mu_{3,3}$

where, $\mathrm{u}_{1}=\mathrm{u}_{\mathrm{x}}, \mathrm{u}_{3}=\mathrm{u}_{\mathrm{z}}$. For $\mathrm{x}$ and $\mathrm{y}$ directions, the stress components after algebraic manipulation read:

$$
\begin{aligned}
& 2 \gamma_{\alpha} \mathrm{A}=\mathrm{Bi}\left(2-\frac{\mathrm{c}^{2}}{\beta^{2}}\right) \\
& -2 \mathrm{~B} \gamma_{\beta}=\mathrm{Ai}\left(2-\frac{\mathrm{c}^{2}}{\beta^{2}}\right)
\end{aligned}
$$

From (17) and (18), the determinant reads:

$$
\left(2-\frac{c^{2}}{\beta^{2}}\right)^{2}-4 \gamma_{\alpha} \gamma_{\beta}=0
$$

where, $\quad \gamma_{\delta}=\sqrt{1-\frac{c^{2}}{\delta^{2}}}$ and $\delta=\alpha, \beta$. Let $\psi=\frac{c^{2}}{\beta^{2}}$ and $\frac{\alpha^{2}}{\beta^{2}}=3$, Eq. 19 becomes:

$$
3 \psi^{3}-24 \psi^{2}+56 \psi-32=0
$$

with roots $\psi_{1}=2-\frac{2}{\sqrt{3}} \approx 0.92, \psi_{2}=4$ and $\psi_{3}=2+\frac{2}{\sqrt{3}}$. For dispersive waves with phase velocity slower than the wave's velocities, only the root $\psi_{1} \approx 0.92$ is valid or $c^{2} \leq \delta^{2}$. Eventually, the Rayleigh waves are dispersive when $\frac{\mathrm{c}}{\beta} \simeq \sqrt{0.92} \simeq 0.9591$. The dispersive stress by dispersive SV waves are supposed similar to $\mathrm{SH}$ waves as illustrated in Fig. 5 since both the SH and SV waves are different in propagation direction only. When SV waves are interfered with $\mathrm{P}$ waves, Rayleigh waves or P-SV waves are formed. It is shown that Rayleigh waves are dispersive when the phase velocity and the velocity of the wave are different or $\mathrm{c}^{2} \leq \delta^{2}$. Dispersion splitting as mentioned by Zhang et al. (2009) will happened when there are two or more travelling SV waves in different velocities. With the significant different of wave's velocities in two different layers of mediums, the dispersion splitting is expected.

The integration of stress components (17) and (18) into the amplitude of Rayleigh wave is important to show the existence of sine and cosine components. The reconstructed stress embedded $\mathrm{P}$ waves and $\mathrm{SV}$ waves are shown by Eq. 20 and 21:

$$
\begin{aligned}
& \mathrm{U}_{\mathrm{P}}=(\operatorname{Biexp}[\mathrm{ik}(\mathrm{ct}-\mathrm{x})])\left[\begin{array}{c}
-\frac{1}{\gamma_{\alpha}}\left(2-\frac{\mathrm{c}^{2}}{\beta^{2}}\right) \exp \left(-\gamma_{\alpha} \mathrm{kz}\right) \\
+\left(\gamma_{\beta}\right) \exp \left(-\gamma_{\beta} \mathrm{kz}\right)
\end{array}\right] \\
& \mathrm{U}_{\mathrm{SV}}=(\operatorname{Bexp}[\mathrm{ik}(\mathrm{ct}-\mathrm{x})])\left[\begin{array}{c}
-\frac{1}{\gamma_{\alpha}}\left(2-\frac{\mathrm{c}^{2}}{\beta^{2}}\right)\left(\gamma_{\alpha}\right) \exp \\
\left(-\gamma_{\alpha} \mathrm{kz}\right)+\exp \left(-\gamma_{\beta} \mathrm{kz}\right)
\end{array}\right]
\end{aligned}
$$

$$
\text { Letting } \mathrm{Q}=(\mathrm{B} \exp [\mathrm{ik}(\mathrm{ct}-\mathrm{x})]), \quad \mathrm{W}=-\frac{1}{\gamma_{\alpha}}\left(2-\frac{\mathrm{c}^{2}}{\beta^{2}}\right)
$$

into (20) and (21), we get:

$$
\left(\frac{\mathrm{U}_{\mathrm{P}}}{\mathrm{Q}}\right)^{2}+\left(\frac{\mathrm{U}_{\mathrm{SV}}}{\mathrm{Q}}\right)^{2}=1
$$

Once Eq. 22 is compared to ellipse equation:

$\sin ^{2}(w t-k x)+\cos ^{2}(w t-k x)=1$

elliptic waves are presented for Rayleigh waves. Apparently, the sine and cosine form of $\mathrm{P}$ waves and SV waves read:

$\mathrm{U}_{\mathrm{P}}=\mathrm{QU}(\mathrm{z}) \sin (\mathrm{wt}-\mathrm{kx})$

$\mathrm{U}_{\mathrm{sv}}=\mathrm{QK}(\mathrm{z}) \cos (\mathrm{wt}-\mathrm{kx}), \mathrm{K}(\mathrm{z})=\gamma_{\alpha} \mathrm{W}(\mathrm{z})$ 


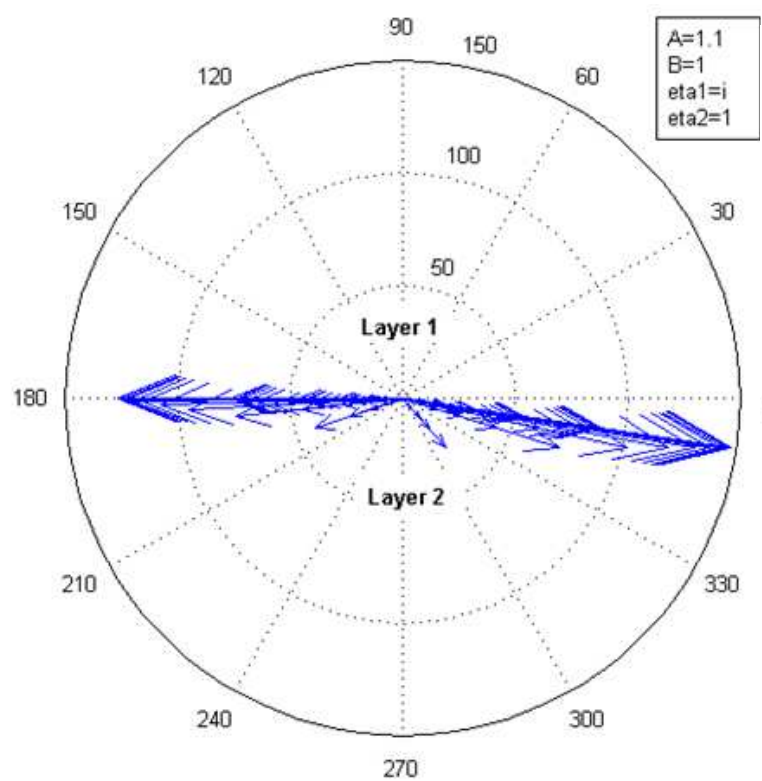

(a)

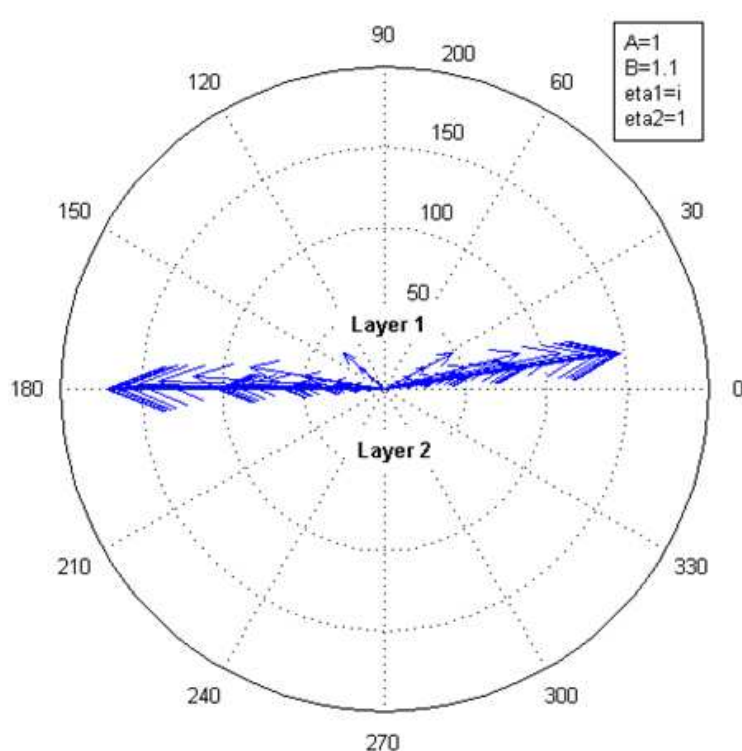

(c)

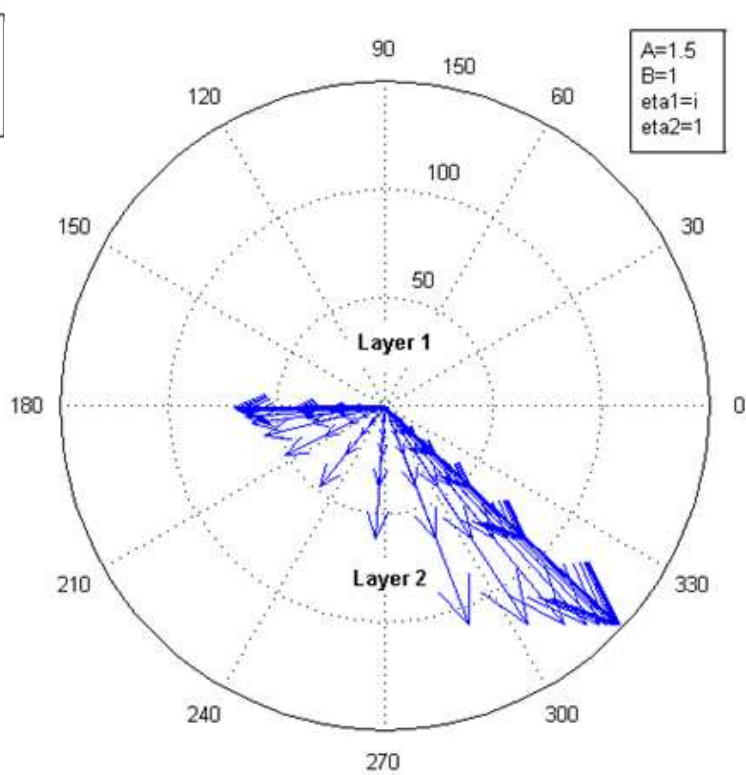

(b)

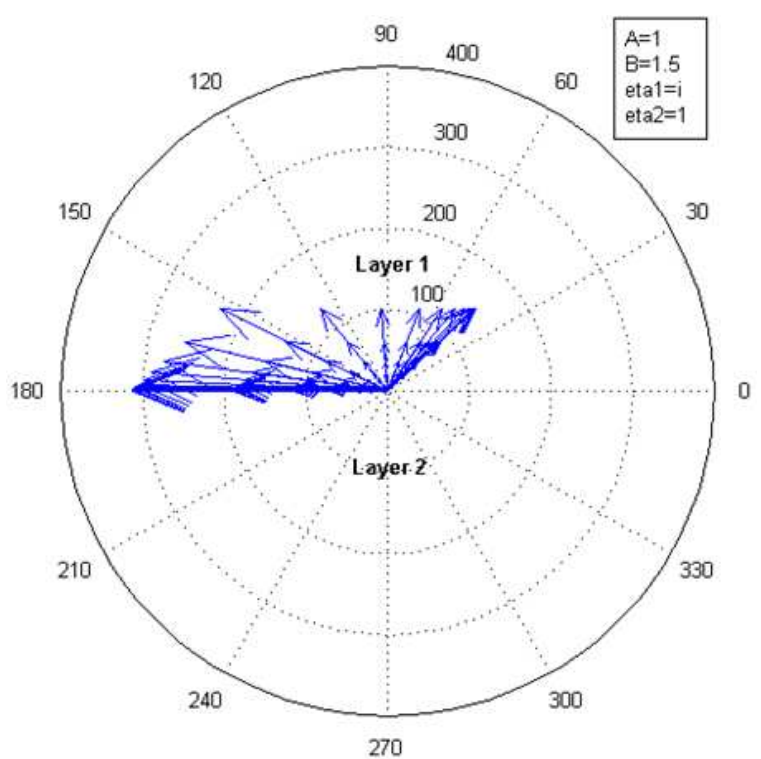

(d)

Fig. 5: The stress by Love waves in between two layers of isotropic mediums. Amplitudes $C_{1}$ and $C_{2}$ are generated by Love waves in Layer 1 and Layer 2 respectively. The diffusive parameters $\eta_{1}$ and $\eta_{2}$ are referred to the Love waves in two different layers of medium

Equation 23 and 24 are the periodic waves. Equation 24 holds cosine component and so the similarity of SV waves and SH waves holding cosine components are shown. Love waves and SV waves are recognized to hold cosine component while Rayleigh waves hold sine and also cosine components. Both sine and cosine components represent different parts of the
Rayleigh waves and also the propagation potential. Since both the SV and SH waves have cosine component, the hauling characteristics should not exist. Rayleigh wave capable of bringing diffusion energy during earthquake is hence blamed to the sine component of $\mathrm{P}$ waves. The interference of $\mathrm{P}$ waves from the Rayleigh waves with the Love waves is 
suggested to carry some of the energy forward as well. Apparently, P and SV waves interfered with SH waves build to 3D elliptic waves when the frequencies allowed.

Besides the sine component of $\mathrm{P}$ waves, there is another possibility for explaining the energy hauling attribute of Rayleigh waves and Love waves. Rayleigh wave is suggested for being conidial waves that do not carry elliptic parameters. Both the waves should hold energy since the stress is adherent.

At the following, the energy equation by Bhatnagar (1979) is exploited to justify the hypothesis. The energy equation reads:

$\frac{1}{2} \mathrm{U}_{\xi}^{2}=\frac{1}{6 \mathrm{k}}(-\mathrm{f}(\mathrm{u}))$

Where:

$f(u)=\left(u-\gamma_{e}\right)\left(u-\beta_{e}\right)\left(\alpha_{e}-u\right)$

The root of the energy Eq. 26 is in according to Fig. 6 .

The energy Eq. 25 can be written as:

$\frac{d u}{\left[\left(u-\gamma_{e}\right)\left(u-\beta_{e}\right)\left(\alpha_{e}-u\right)\right]^{1 / 2}}=\frac{d \xi}{(3 K)^{1 / 2}}$

Letting $p^{2}=\alpha_{e}-u, \quad \alpha_{e}-\beta_{e}=\frac{p^{2}}{q^{2}}, \quad s^{2}=\frac{\alpha_{e}-\beta_{e_{e}}}{\alpha_{e}-\gamma_{e}}$, $q=\frac{p}{\sqrt{\alpha_{e}-\beta_{e}}}=\sqrt{\frac{\alpha_{e}-u}{\alpha_{e}-\beta_{e}}}$ into (27), we get:

$$
\begin{aligned}
\xi & =\sqrt{\frac{12 \mathrm{~K}}{\alpha_{\mathrm{e}}-\gamma_{\mathrm{e}}}} \int_{0}^{\mathrm{q}} \frac{\mathrm{d}_{\mathrm{q}}}{\left[\left(1-\mathrm{s}^{2} \mathrm{q}^{2}\right)\left(1-\mathrm{q}^{2}\right)\right]^{1 / 2}} \\
& =\sqrt{\frac{12 \mathrm{~K}}{\alpha_{\mathrm{e}}-\gamma_{\mathrm{e}}}} \operatorname{Sn}^{-1}(\mathrm{q}, \mathrm{s})
\end{aligned}
$$

Or:

$\mathrm{u}(\xi)=\beta+(\alpha-\beta) \mathrm{Cn}^{2}\left[\xi \sqrt{\frac{\alpha_{\mathrm{e}}-\gamma_{\mathrm{e}}}{12 \mathrm{~K}}}, \mathrm{~s}\right]$

Equation 29 is the Cnoidal waves extracted from energy equation where $\mathrm{Sn}$ and $\mathrm{Cn}$ are the Jacobian Elliptic functions. $\mathrm{s}$ is the elliptic parameter to decide the troughs of the Cnoidal waves.

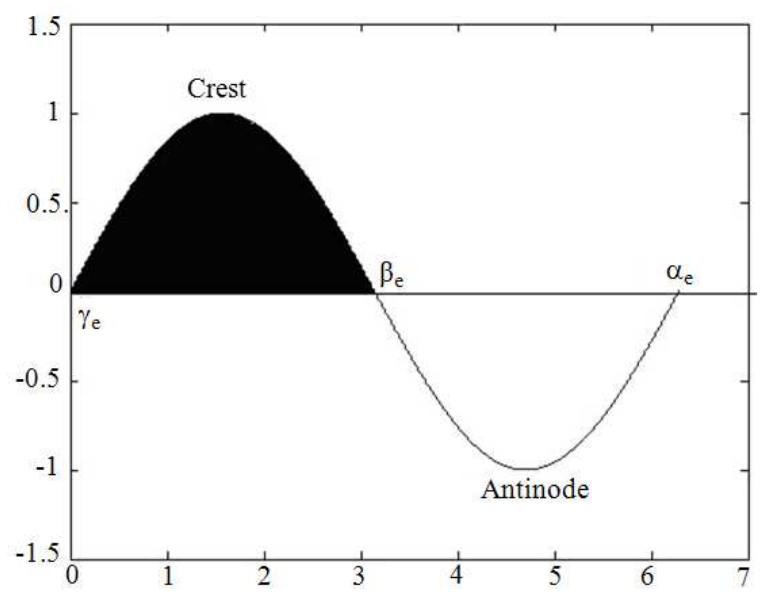

Fig. 6: The root of the energy equation

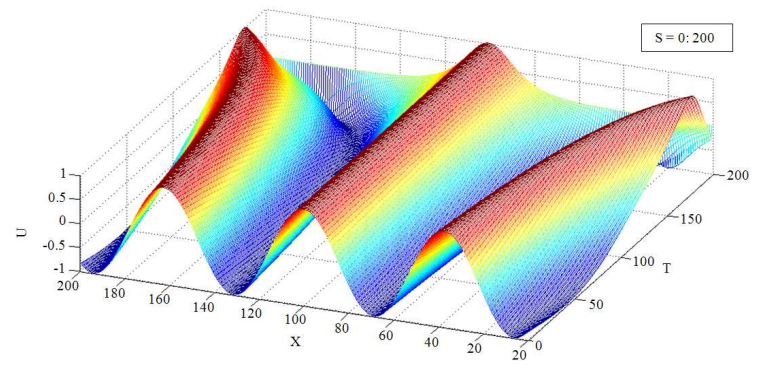

Fig. 7: The transformation of Rayleigh waves to conidial waves with the increment of elliptic parameter, $\mathrm{s}$ from 0 to 200 with time $t$.

Referring to Fig. 7 it is evidenced that the troughs of the waves have changed when the elliptic parameter is increasing. Without the elliptic parameters, the conical wave is simply a sinusoidal wave or acoustic wave that is similar to Rayleigh wave. In fact, these nonlinear waves are capable of carrying and transporting vast energy.

\section{RESULTS AND DISCUSSION}

The cosine component explained the capability of shear waves carrying energy. Love waves which are similar to standing waves do not carry energy forward once the standing waves are defined as the interference of two waves moving in opposite direction. High diffusion energy is expected at the rupturing path with the energy diffused when they arrive at medium surface. However, the near surface stress and the stress by interfered Love waves near to the surface might differ given that the diffusive terms are considered for the stress component of the interfered waves. 
Overlapped amplitude is shown by interfered waves. From the discussions, the shear waves either SV or SH waves have tendency for being interfered by the $\mathrm{P}$ waves which are part of the Rayleigh waves. Rayleigh waves only consist of sine and cosine component. If the cosine component is proven for unable to carry energy forward, then the sine component is responsible for propagating the energy. The vein structure found after an earthquake illustrates the shear waves or SV waves can propagate in the isotropic medium. Through 3D particle motion, it is suggested that these SV waves and Love waves are brought forward by the $\mathrm{P}$ waves which are belong to Rayleigh waves. There is another possibility for energy hauling during earthquake. The Cnoidal waves which are also known as nonlinear periodic waves are differed by elliptic parameter. When the elliptic parameter is too small, the Cnoidal waves should look alike seismic waves for which the self reinforced capability of these nonlinear waves propagate energy during an earthquake.

Recently, the amplification of linear strain in a layer excited by a shear wave earthquake pulses are found by Gicev and Trifunac (2010). The interference of two waves in opposite directions is exploited and the amplification is due to sine pulse. The simulated figures by Gicev and Trifunac (2010) illustrate flat troughs similar to conical waves as well. Hence, it is well said the sine component of Rayleigh waves are propagating the energy of interfered Love waves. Ability of Conidial waves interfering the shear waves are suggested for further study.

\section{CONCLUSION}

Sine components of Rayleigh waves are responsible for propagating the cosine components. The energy or stress relating to the cosine component does not carry the energy forward. There is another likelihood for explaining the incident of energy hauling during an earthquake. Cnoidal waves with low elliptic parameters are similar to Rayleigh waves and the self reinforced capabilities of these nonlinear waves are known for carrying energy.

\section{REFERENCES}

Ari-Menahem, B. and S.J. Singh, 1981. Seismic Waves and Sources. 2nd Edn., Springer-Verlag, New York, pp: 1136. ISBN: 0-486-40461-7

Bhatnagar, P.L., 1979. Nonlinear Waves in One Dimensional Dispersive System. 1st Edn., Thomson Press Limited, India, pp: 142. ISBN: 0198535317
Brothers, R.J., A.E.S. Kemp and A.J. Maltman, 1996. Mechanical development of vein structures due to the passage of earthquake waves through poorly consolidated sediments. Tectonophysics, 260: 227-244. DOI: 10.1016/0040-1951(96)00088-1

Dauxious, T. and M. Peyrard, 2006. Physics of Solitons. 1st Edn., Cambridge University Press, United Kingdom, pp: 422. http://www.amazon.com/gp/search?index=books\&l inkCode $=$ qs\&keywords $=0521854210$

Drazin, P.G. and R.S. Johnson, 1989. Solitons: An introduction. 1st Edn., Cambridge University Press, United Kingdom, pp: 226. ISBN: 0-521-33655-4

Gicev, V. and M.D. Trifunac, 2010. Amplification of linear strain in a layer excited by a shear wave earthquake pulse. Soil Dyn. Earthq. Eng., 30: 1073-1081. DOI: 10.1016/J.SOILDYN.2010.04.018

Ohsumi, T. and Y. Ogawa, 2008. Vein structures, like ripple marks, are formed by short wavelength shear waves. J. Struct. Geol., 30: 719-724. DOI: 10.1016/J.JSG.2008.02.002

Ribal, A., 2007. Numerical study on the effect of linear friction of long-wave propagation and breaking with sloping bottom. J. Math. Stat., 3: 263-267. DOI: $10.3844 / .2007 .263 .267$

Ribal, A., 2008. Numerical study of tsunami waves with sloping bottom and nonlinear friction. J. Math. Stat., 4: 41-45. DOI: 10.3844/.2008.41.45

Zhang, S.X., Y. Wang, H.W. Zhou and L.S. Chan, 2009. Dispersion splitting of Rayleigh waves in layered azumuthally anisotropic media. J. Applied Geophys., $\quad$ 67: 130-142. DOI: 10.1016/J.JAPPGEO.2008.10.008 